Einführung in das Schwerpunktthema

\title{
Geteilte Verantwortung im Verbraucherschutz
}

I Von Ulla Simshäuser $\mathrm{n}$ die deutsche Verbraucherpolitik ist Bewegung gekommen. Zwei wichtige Meilensteine sind das vor gut zwei Jahren institutionalisierte Bundesministerium für Verbraucherschutz, Ernährung und Landwirtschaft (BMVEL) und die Veröffentlichung des Verbraucherpolitischen Aktionsplans im Mai diesen Jahres. Bereits Ende 2002 konstituierte sich der wissenschaftliche Beirat des Ministeriums und im April 2003 hat er der Ministerin sein Diskussionspapier zur Verbraucher- und Ernährungspolitik überreicht. So wichtig politische Schwerpunkte und Orientierungen sind, so ist trotzdem klar, dass angesichts abnehmender nationalstaatlicher Steuerungskapazitäten und zunehmender Pluralisierung von wirtschaftsrelevanten Akteuren und Lebensstilen ein wirksamer Verbraucherschutz in der praktischen Umsetzung auf die Kooperation und geteilte Verantwortungsnahme einer Vielzahl von gesellschaftlichen Interessensgruppen angewiesen ist. Dem entspricht, dass neue Politiken für die Praxis nicht verordnet, sondern nur ausgehandelt werden können

Das Prinzip der Vorsorge und Verantwortung für Verbraucherschutz spielt auf nationaler wie europäischer Ebene eine große Rolle. Die folgenden Beiträge fragen, welche Akteure im Sinne einer geteilten Verantwortung zum Handeln aufgerufen sind. Sie präsentieren Positionen zentraler Akteure sowie Chancen und Grenzen von Risikokommunikation und Verbraucherinformation durch Unternehmen. Sie werden durch konzeptionelle Überlegungen ergänzt, die Verbraucherpolitik in den Kontext von Gesundheits- und Sozialpolitik sowie Geschlechtergerechtigkeit stellen.

\section{- Die Beiträge im Einzelnen}

Lucia Reisch gibt zunächst einen ersten Überblick über die ersten Schritte des BMVEL hin zu einem „New Deal“ des Verbraucherschutzes. Sie skizziert Politikelemente auf nationaler, europäischer und globaler Ebene, die Vorsorge zum Schutz des Verbrauchers nicht nur als gesundheitliche Risikovorsorge, sondern als ,Zukunftsvorsorge im Sinne einer, sozial, ökologisch und öko- nomisch nachhaltigen Gesellschaftsentwicklung und einer beide Marktseiten fördernden Standortpolitik" begreifen.

Ein hohes Schutzniveau von Verbrauchern stärken, so Matthias Berninger, liegt im zentralen Verantwortungsbereich des Staates. Dies wird insbesondere durch ein hohes Maß an Markttransparenz durch Produktinformation gefördert, die Nachhaltigkeit des Konsums durch das Instrument des nachhaltigen Warenkorbs. Ob und in wieweit nicht nur staatliche sondern auch private Akteuren glaubwürdig Verbraucherinformationen weitergeben können oder ob hier nicht, ,der Bock zum Gärtner" gemacht wird, fragen sich Ulf Schrader, Ingo Schönheit und Ursula Hansen. Die Bereitstellung detaillierter Produktinformation durch Unternehmen ist vergleichsweise kostengünstig möglich, in bestimmten Bereichen kann die Arbeit von Behörden zum Beispiel durch Warnhinweise aktiv unterstïtzt werden und für das Unternehmen können Wettbewerbsvorteile bei bestimmten Verbrauchergruppen entstehen. Andererseits stellt sich die Frage der Glaubwürdigkeit und der Motive der zur Verfügung gestellten Informationen. Es wird daher immer Bereiche geben, so ihr Fazit, in denen staatliche Eingriffe zur Informationsregelung unerlässlich sind.

Nachhaltiger Konsum ist nur möglich, wenn VerbraucherInnen über die entsprechenden Implikationen beim Kauf informiert werden. Durch eine verschleiernde Preispolitik können sie, so die implizite Aussage des Beitrags von Thilo Bode, den Markt gar nicht über ihr Konsumverhalten rational steuern. Preise, wie der von Fleisch, müssen die tatsächlichen Kosten widerspiegeln. Solange keine Markttransparenz über tatsächliche Kosten existiert, werden VerbraucherInnen letztlich unwissend und unmündig gehalten und können nicht nachhaltig konsumieren.

Der Verbraucherresonanz auf Umweltzeichen geht Dirk Scheer nach. Ergebnisse einer international vergleichenden Erhebung des Instituts für ökologische Wirtschaftsforschung (IÖW) haben gezeigt, dass VerbraucherInnen nur eine begrenzte Zahl von Umweltkennzeichen kennen und die Produktkennzeichnung auch von vielen Unternehmen als nicht attraktiv empfunden wird. Dieser „Desillusionierung" stehen aber neuerliche Anreize auf europäischer Ebene durch die Entwicklung einer integrierten Produktpolitik entgegen.

Verbraucherinformation und Produktkennzeichen gehören zum klassischen Repertoire des Verbraucherschutzes und gelten als Voraussetzung dafür, dass VerbraucherInnen sich rational, mündig und kompetent verhalten können. Der Beitrag von Irmgard Schultz weist indes darauf hin, dass Männer und Frauen ganz unterschiedliche Zugänge zu Information haben und dass verbraucherpolitische Instrumente und Politiken unterschiedlich auf die soziale Stellung der Geschlechter wirken. Verbraucherpolitische Maßnahmen müssen deshalb immer hinsichtlich ihrer Auswirkungen auf Geschlechtergerechtigkeit geprüft und bereits bei der Planung einem Gender Mainstreaming unterzogen werden. Dabei müssen drei Elemente immer gepruift werden: Einflüsse auf die geschlechtsspezifische Arbeitsteilung, ihre Wirkungen auf die gesellschaftliche Organisation von Intimität (zum Beispiel Gesundheit und Reproduktion) und auf die unterschiedliche Gestaltungsmacht von Männern und Frauen.

Nicht nur Gender, auch Armut muss bei der Frage der Verantwortung für Verbraucherschutz zum Thema werden. Der Beitrag von Frank Waskow, Regine Rehaag und Eva Barlösius weist auf die zunehmende Ernährungsarmut unter VerbraucherInnen und die völlig unzureichende Datenlage für die Entwicklung zielgruppenspezifischer Präventionsmaßnahmen hin. Ulla Simshäuser diskutiert schließlich für den gesundheitsbezogenen Verbraucherschutz die Notwendigkeit, angesichts der gesellschaftlichen Herausforderungen einerseits und steigender Gesundheitsausgaben andererseits, den gesundheitsbezogenen Verbraucherschutz um die Dimension der Gesundheitsförderung und die Stärkung der gesundheitlichen Kompetenz von VerbraucherInnen zu erweitern.

\section{Die Autorin}

Ulla Simshäuser ist wissenschaftliche Mitarbeiterin in den Forschungsfeldern ökologische Produktpolitik und ökologischer Konsum des Instituts für ökologische Wirtschaftsforschung (IÖW).

Kontakt: IÖW-Regionalbüro Baden-Württemberg, Bergstraße 7, 69120 Heidelberg. Tel. 06221-649160, E-Mail: Ulla.Simshaeuser@heidelberg.ioew.de 
(c) 20I0 Authors; licensee IÖW and oekom verlag. This is an article distributed under the terms of the Creative Commons Attribution Non-Commercial No Derivates License (http://creativecommons.org/licenses/by-nc-nd/3.o/), which permits unrestricted use, distribution, and reproduction in any medium, provided the original work is properly cited. 